\title{
NODAL DOMAIN THEOREMS AND BIPARTITE SUBGRAPHS*
}

\author{
TÜRKER BIYIKOĞLU ${ }^{\dagger}$, JOSEF LEYDOLD ${ }^{\ddagger}$, AND PETER F. STADLER ${ }^{\S}$
}

\begin{abstract}
The Discrete Nodal Domain Theorem states that an eigenfunction of the $k$-th largest eigenvalue of a generalized graph Laplacian has at most $k$ (weak) nodal domains. We show that the number of strong nodal domains cannot exceed the size of a maximal induced bipartite subgraph and that this bound is sharp for generalized graph Laplacians. Similarly, the number of weak nodal domains is bounded by the size of a maximal bipartite minor.
\end{abstract}

Key words. Graph Laplacian, Nodal Domain Theorem, Eigenvectors, Bipartite Graphs

AMS subject classifications. 05C50 Graphs and matrices, 05C22 Signed, gain and biased graphs, 05C83 Graph minors.

1. Introduction. The eigenfunctions of elliptic differential equations of the form $L[u]+\lambda \rho u=0,(\rho>0)$, on a domain $D$ with arbitrary homogeneous boundary conditions have an interesting geometric property: if the eigenfunctions are ordered according to increasing eigenvalues, then the nodes of the $n$th eigenfunction $u_{n}$ divide the domain into no more than $n$ subdomains $[7$, Chap.6, §6]. No assumptions are made about the number of independent variables. These sub-domains have since become known as nodal domains, see e.g. [4].

The discrete analogue of a "nodal domain" is a connected set of vertices, i.e., a connected subgraph of a graph $G$, on which the eigenvector has the same, strict or loose, sign. Of course, such a set of vertices is not "bounded" by "nodes"; it is merely "bounded" by vertices of the opposite loose sign. An more appropriate name for such an entity would thus appear to be sign graph [8]. We nevertheless use here the established terminology from the manifold case. The discrete analogues of the elliptic differential operator $L[u]$ are a certain class of symmetric matrices that reflect the structure of underlying graph $G$, so-called (generalized) graph Laplacians [6, 5, 12].

In general, generalized graph Laplacians satisfy an analog of Courant's Nodal Domain Theorem [8]. As in the case of manifolds, it is of interest to consider special classes of graphs for which stronger and more detailed results, e.g. tighter bounds on the number of nodal domains, could be derived. For some results on trees and hypercubes we refer to $[1,2,9]$. In this letter we consider bipartite graphs and upper bounds on the number of nodal domains that are determined by the structure of the underlying graph itself.

\footnotetext{
* Received by the editors on ... Accepted for publication on .... Handling Editor: ...

$\dagger$ Max Planck Institute for Mathematics in the Sciences, Inselstrasse 22-26, D-04103 Leipzig, Germany. biyikoglu@mis.mpg.de,

${ }^{\ddagger}$ Dept. of Statistics and Mathematics. University of Economics and Business Administration, Augasse 2-6, A-1090 Wien, Austria. leydold@statistik.wu-wien.ac.at

$\S$ Bioinformatics Group (Department of Computer Science) and Interdisciplinary Center for Bioinformatics, University of Leipzig, Härtelstrasse 16-18, D-04107 Leipzig, Germany; Institute for Theoretical Chemistry, University of Vienna, Währingerstrasse 17, A-1090 Wien, Austria; The Santa Fe Institute, 1399 Hyde Park Rd., Santa Fe NM 87501. studla@bioinf.uni-leipzig.de
} 
We briefly recall the construction of generalized graph Laplacians, derive a few useful properties, and formally introduce strong and weak nodal domains in the following two sections. We then offer an alternative proof of a theorem by Roth demonstrating that the largest eigenvalue of a graph Laplacian of a bipartite graph always has as many nodal domains as vertices. The main results of this letter finally show that bipartite induced subgraphs and bipartite minors can provide non-trivial bounds on the number of nodal domains. In the cases of bipartite induced subgraphs we show that the bound cannot be improved.

2. Generalized Graph Laplacians. Let $G(V, E)$ be a simple graph with vertex set $V$ and edge set $E$. We use the convention that $|V|=n$ and $|E|=m$, i.e., $G$ is a graph with $n$ vertices and $m$ edges. The Laplacian of $G$ is the matrix

$$
\mathbf{L}(G)=\mathbf{D}(G)-\mathbf{A}(G)
$$

where $\mathbf{D}(G)$ is the diagonal matrix whose entries are the degrees of the vertices of $G$, i.e. $D_{v v}=d(v)$, and $\mathbf{A}(G)$ denotes the adjacency matrix of $G$. For the function $\mathbf{L} f$ we find

$$
(\mathbf{L} f)(x)=\sum_{y \sim x}[f(x)-f(y)]=d(x) f(x)-\sum_{y \sim x} f(y) .
$$

We find this "functional" notation more convenient than using vectors indexed by vertices. In particular, it simplifies comparison with analogous results on manifolds. The quadratic form of the graph Laplacian can be computed via Green's formula as

$$
\langle f, \mathbf{L} f\rangle=\sum_{x, y \in V} L_{x y} f(x) f(y)=\sum_{x y \in E}(f(x)-f(y))^{2} .
$$

This equality immediately shows that the graph Laplacian is a nonnegative operator, i.e., all eigenvalues are greater than or equal to 0.

The graph Laplacian $\mathbf{L}$ can be generalized in following way, see [6]: A symmetric matrix $\mathbf{M}(G)$ is called a generalized Laplacian (or discrete Schrödinger operator) of $G$ if it has nonpositive off-diagonal entries and for $x \neq y, M_{x y}<0$ if and only if the vertices $x$ and $y$ are adjacent. On the other hand, for each symmetric matrix with nonpositive off-diagonal entries there exists a graph where two distinct vertices $x$ and $y$ are adjacent if and only if $M_{x y}<0$. Similarly to (2.2) we have

$$
(\mathbf{M} f)(x)=\sum_{y \sim x}\left(-M_{x y}\right)[f(x)-f(y)]+p(x) f(x),
$$

where $p(x)=M_{x x}+\sum_{y \sim x} M_{x y}$. The function $p(x)$ can be viewed as a potential defined on the vertices. Defining a matrix $\mathbf{W}$ consisting of $W_{x y}=M_{x y}$ for $x \neq y$ and $W_{x x}=-\sum_{y \neq x} M_{x y}$ and a diagonal matrix $\mathbf{P}$ with the potentials $p(x)$ as its entries we can decompose every generalized Laplacian as

$$
\mathbf{M}=\mathbf{W}+\mathbf{P} .
$$


The matrix $\mathbf{W}$ can be seen as discrete elliptic operator. The quadratic form of the generalized Laplacian can then be computed as

$$
\begin{aligned}
\langle f, \mathbf{M} f\rangle & =\sum_{x y \in E}\left(-M_{x y}\right)(f(x)-f(y))^{2}+\sum_{x \in V} p(x) f(x)^{2} \\
& =\sum_{x \in V} M_{x x} f(x)^{2}+2 \sum_{x y \in E} M_{x y} f(x) f(y) .
\end{aligned}
$$

The following remarkable result for the eigenvalues of a generalized Laplacian can be easily derived.

TheOREM 2.1. Let $\lambda$ be an eigenvalue of a generalized Laplacian $\mathbf{M}=\mathbf{W}+\mathbf{P}$ with eigenfunction $f$. Then either $\sum_{v \in V} f(v)=\sum_{v \in V} p(v) f(v)=0$, or

$$
\lambda=\frac{\sum_{v \in V} p(v) f(v)}{\sum_{v \in V} f(v)} .
$$

Proof. Let $\mathbf{1}=(1, \ldots, 1)^{\top}$. Then a straightforward computation gives

$$
\begin{aligned}
\langle\mathbf{1}, \mathbf{M} f\rangle & =\sum_{v \in V}\left(\sum_{w \sim v}\left(-M_{v w}\right)(f(v)-f(w))+p(v) f(v)\right) \\
& =\sum_{v, w \in V}\left(-M_{v w}\right)(f(v)-f(w))+\sum_{v \in V} p(v) f(v) \\
& =\sum_{v, w \in V} M_{v w} f(w)-\sum_{v, w \in V} M_{v w} f(v)+\sum_{v \in V} p(v) f(v) \\
& =\sum_{v \in V} p(v) f(v) .
\end{aligned}
$$

Since $f$ is an eigenfunction we find $\langle\mathbf{1}, \mathbf{M} f\rangle=\lambda \sum_{v \in V} f(v)$, and thus the proposition follows. $\mathrm{Q}$

REMARK 2.2. The case $\sum_{v \in V} f(v)=0$ happens, for example, for all eigenfunctions to an eigenvalue $\lambda>\lambda_{1}$ when the eigenfunction $f_{1}$ to $\lambda_{1}$ is constant. This is the case if and and only if $p(v)$ is constant for all $v \in V$.

REMARK 2.3. Theorem 2.1 has a more delicate form for the Dirichlet Eigenvalues of a graph with boundary. There some of the vertices are considered as "boundary" and the Dirichlet operator is the Laplacian restricted to the interior (non-boundary) vertices of the graph, i.e., where the corresponding rows and columns of the Laplacian matrix are removed [10]. Dirichlet operators can be seen as the discrete analogs of Dirichlet eigenvalue problems on bounded manifolds. Denote the number of boundary vertices adjacent to some vertex $v$ by b(v). Then, by Thm. 2.1, we find [3]:

$$
\lambda=\frac{\sum_{v \in V} b(v) f(v)}{\sum_{v \in V} f(v)} .
$$

3. Nodal Domains. Consider an a graph $G$ and an arbitrary function $f$ : $V(G) \rightarrow \mathbb{R}$ defined on its vertex set. A positive (negative) strong nodal domain of $f$ is a maximal connected induced subgraph $H$ of $G$ such that $f(v)>0(f(v)<0)$ holds for all $v \in V(H)$. In contrast, a positive (negative) weak nodal domain of $f$ is a maximal connected induced subgraph $H$ of $G$ such that $f(v) \geq 0(f(v) \leq 0)$ for 
all $v \in V(H)$ and there is at least one non-zero vertex, i.e., there is a $w \in V(H)$ for which $f(w) \neq 0$. In the following we will be interested in the number of strong and weak nodal domains of a function $f$ which we denote by $\mathfrak{S}(f)$ and $\mathfrak{W}(f)$, respectively. Obviously, $\mathfrak{W}(f) \leq \mathfrak{S}(f)$.

The obvious difference between the definitions of strong and weak nodal domains is the rôle of zero vertices, i.e. vertices where the function $f$ vanishes. While such vertices separate positive (or negative) strong nodal domains, they join weak nodal domains. In fact, each zero vertex simultaneously belongs to exactly one weak positive nodal domain and exactly one weak negative nodal domain. If two different weak nodal domains $D_{1}$ and $D_{2}$ overlap, then they must have opposite signs except on zero vertices. In the following we will only consider nodal domains of an eigenfunction of a generalized Laplacian.

We focus our attention on the $k$-th eigenvalue $\lambda_{k}$ with multiplicity $r$ of a generalized Laplacian M. We assume throughout this paper that the eigenvalues are labeled in ascending order starting with 1 , so that

$$
\lambda_{1} \leq \cdots \leq \lambda_{k-1}<\lambda_{k}=\lambda_{k+1}=\cdots=\lambda_{k+r-1}<\lambda_{k+r} \leq \cdots \leq \lambda_{n} .
$$

An eigenfunction of $\mathbf{M}$ affording $\lambda_{k}$ will be denoted by $f_{k}$. These conventions allow us to formulate discrete versions of Courant's Nodal Domain Theorem in a compact way.

Theorem 3.1 (Discrete Nodal Domain Theorem, [8]). Let $\mathbf{M}$ be a generalized Laplacian of a connected graph with $n$ vertices. Then any eigenfunction $f_{k}$ corresponding to the $k$-th eigenvalue $\lambda_{k}$ with multiplicity $r$ has at most $k$ weak nodal domains and $k+r-1$ strong nodal domains:

$$
\mathfrak{W}\left(f_{k}\right) \leq k \quad \text { and } \quad \mathfrak{S}\left(f_{k}\right) \leq k+r-1 .
$$

4. Bipartite Graphs. Let us first consider the largest eigenvalue of a connected bipartite graph $G$. We start by providing an alternative proof of a result by R. Roth:

THEOREM 4.1 ([14]). Let $G\left(V_{1} \cup V_{2}, E\right)$ be a connected bipartite graph with $n=\left|V_{1} \cup V_{2}\right|$ vertices and let $\mathbf{M}$ be a generalized Laplacian of $G$. Then there is an eigenfunction $f$ to the largest eigenvalue of $\mathbf{M}$, such that $f$ is positive on $V_{1}$ and negative on $V_{2}$ or vice versa and hence satisfies $\mathfrak{W}(x)=\mathfrak{S}(x)=n$.

Proof. The largest eigenvalue $\lambda_{n}$ of $\mathbf{M}$ is determined by the maximum of the Rayleigh quotient $\mathcal{R}_{\mathbf{M}}(f)=\langle f, \mathbf{M} f\rangle /\langle f, f\rangle$. We may assume that $f$ is normalized so that $\langle f, f\rangle=1$; thus by (2.7) we have

$$
\mathcal{R}_{\mathbf{M}}(f)=\sum_{x \in V} M_{x x} f(x)^{2}+2 \sum_{x y \in E} M_{x y} f(x) f(y) .
$$

Let $f_{n}$ be an eigenfunction affording $\lambda_{n}$ and define $g(x)=\left|f_{n}(x)\right|$ if $x \in V_{1}$ and $g(x)=-\left|f_{n}(x)\right|$ if $x \in V_{2}$. We have $\mathcal{R}_{\mathbf{M}}(g) \geq \mathcal{R}_{\mathbf{M}}\left(f_{n}\right)$; this inequality is strict if and only if there is an edge $x y \in E$ such that $f_{n}(x) f_{n}(y)>0$. Since $f_{n}$ maximizes $\mathcal{R}_{\mathbf{M}}$ we have $f_{n}(x) f_{n}(y) \leq 0$ for all $x y \in E$. Therefore $g$ is an eigenfunction of $\lambda_{n}$. 


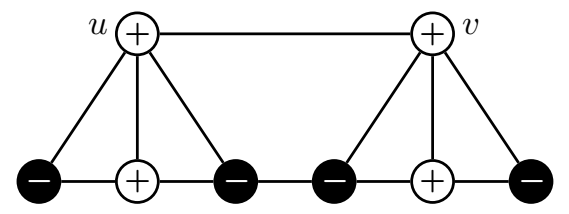

FIG. 5.1. Sign pattern of an eigenfunction to the Laplacian $\mathbf{L}$ with maximal number of strong nodal domains: $\mathfrak{S}(f)=5<6=|V(H)|$. One easily checks that for all simple eigenvalues there are at most 4 strong nodal domains. For the only multiple eigenvalue $\lambda_{5}=4$ (multiplicity $r=2$ ) we have $f(u)=f(v)$. If both are nonzero $\mathfrak{S}(f) \leq 4$; otherwise we have 5 strong nodal domains.

Now suppose $g(x)=0$ for some $x \in V_{1}$. Then $\sum_{y \sim x} M_{x y} g(y)=\lambda_{n} g(x)=0$. Since all neighbors of $x$ are contained in $V_{2}$ this implies $g(y)=0$ for all $y \sim x$. Repeating the argument shows that $g$ must vanish, a contradiction to $\langle g, g\rangle=1$. Thus $g(x)>0$ and hence either $f_{n}=g$ or $f_{n}=-g$. Since any two neighboring vertices have opposite strict signs, we see that each vertex $x \in V$ is a strong nodal domain, and the theorem follows. $\mathrm{Q}$

The Discrete Nodal Domain Theorem now directly implies another result from [14]:

COROLLARY 4.2. The largest eigenvalue of a generalized Laplacian of a connected bipartite graph is simple.

Proof. By Thm. 3.1 any eigenfunction to eigenvalue $\lambda_{n-1}$ has at most $n-1$ weak nodal domains. If the largest eigenvalue is not simple, i.e., $\lambda_{n-1}=\lambda_{n}$, then the eigenfunction of Thm. 4.1 would also be an eigenfunction to $\lambda_{n-1}$, a contradiction.

5. Bipartite Subgraphs and Minors. Obviously, a graph $G$ cannot have $n$ nodal domains unless it is bipartite. This suggests to use maximal bipartite subgraphs as a way of constructing bounds on the maximal number of nodal domains. Indeed, we have

THEOREM 5.1. Let $G(V, E)$ be a connected graph and $H$ be an induced bipartite subgraph of $G$ with maximum number of vertices. Then for any eigenfunction $f$ of a generalized Laplacian $\mathbf{M}(G), \mathfrak{S}(f) \leq|V(H)|$.

Proof. We delete all zero vertices and for each strong nodal domain we delete all but one vertex. The subgraph $H^{\prime}$ induced by the remaining vertices is bipartite and has $\left|V\left(H^{\prime}\right)\right|=\mathfrak{S}(f)$ vertices. By construction, $\left|V\left(H^{\prime}\right)\right| \leq|V(H)|$, and the result follows. $\square$

Unfortunately, finding a maximal induced bipartite subgraph of $G$ is a well known NP-complete problem, see, e.g., [11]. In general, the upper bound of Thm. 5.1 is not sharp for the graph Laplacian L, see Fig. 5.1 for a counterexample. However, we can show that the bound of Thm. 5.1 is sharp for generalized Laplacians of every given graph.

THEOREM 5.2. Let $G$ be a connected graph and $H$ be a maximal induced bipartite subgraph of $G$, then there exists a generalized Laplacian $\mathbf{M}(G)$ such that $\mathbf{M}(G)$ has an eigenfunction $f$ with $|V(H)|$ strong nodal domains.

Proof. Let $H$ be a maximum induced bipartite subgraph of $G$ with components $C_{1}, \ldots, C_{k}$ and let $R$ be the set of remaining vertices of $G$. Let $\mathbf{M}_{1}, \ldots, \mathbf{M}_{k}$ be 
generalized Laplacians of $C_{1}, \ldots, C_{k}$ such that diagonal elements of $\mathbf{M}_{i}$ are positive. By Thm. 4.1 the largest eigenvalue $\mu_{i}$ of $\mathbf{M}_{i}$ has an eigenfunction $f_{i}$ with $\mathfrak{S}\left(f_{i}\right)=$ $\left|V\left(C_{i}\right)\right|$. The eigenvalues $\mu_{i}$ are positive, since $\operatorname{Tr}\left(\mathbf{M}_{i}\right)>0$. Thus we can assume without loss of generality that $\mu_{i}=1$ (otherwise replace $\mathbf{M}_{i}$ by $\frac{1}{\mu_{i}} \mathbf{M}_{i}$ ). We now define a generalized Laplacian for $G$ by

$$
\mathbf{M}=\left(\begin{array}{ccccc}
\mathbf{M}_{1} & 0 & \cdots & 0 & \mathbf{B}_{1}^{\top} \\
0 & \mathbf{M}_{2} & \cdots & 0 & \mathbf{B}_{2}^{\top} \\
\vdots & \vdots & \ddots & \vdots & \vdots \\
0 & 0 & \cdots & \mathbf{M}_{k} & \mathbf{B}_{k}^{\top} \\
\mathbf{B}_{1} & \mathbf{B}_{2} & \cdots & \mathbf{B}_{k} & \mathbf{M}_{R}
\end{array}\right)
$$

where $\mathbf{M}_{R}$ is some generalized Laplacian on the graph induced by $R$, and the $\mathbf{B}_{i}$ matrices with nonpositive entries. Notice that each vertex $v \in R$ has (at least) two neighbors $w_{1}$ and $w_{2}$ in some $C_{j}$ such that $f_{j}\left(w_{1}\right)$ and $f_{j}\left(w_{2}\right)$ have opposite (strict) sign, since otherwise we could construct a new bipartite graph with more vertices then $H$. Thus we can choose $\mathbf{B}_{1}, \ldots, \mathbf{B}_{k}$ such that $\mathbf{B}_{1} f_{1}(v)+\cdots+\mathbf{B}_{k} f_{k}(v)=0$. Now construct a function $f$ by $f(v)=f_{i}(v)$ if $v \in C_{i}$ and $f(v)=0$ if $v \in R$. Then a straightforward computation gives $(\mathbf{M} f)(v)=f_{i}(v)=f(v)$ if $v \in C_{i}$ and $(\mathbf{M} f)(v)=\left(\mathbf{B}_{1} f_{1}+\cdots+\mathbf{B}_{k} f_{k}\right)(v)=0=f(v)$ if $v \in R$. Hence $\mathbf{M} f=f$ and $f$ is an eigenfunction with $\sum_{i=1}^{k}\left|V\left(C_{i}\right)\right|=|V(H)|$ nodal domains.

REMARK 5.3. Theorem 5.2 suggests to use eigenfunctions of (randomly generated) generalized Laplacians as a means of constructing approximate solutions of the maximum induced subgraph problem. However, the performance of such an approach for large graphs is an open problem and has to be studied by means of computational experiments.

The obvious alternative to considering induced subgraphs is to investigate graph minors. This yields a corresponding upper bound for the number of weak nodal domains of an arbitrary function.

TheOREM 5.4. Let $G(V, E)$ be a connected graph and $G^{*}=\left(V^{*}, E^{*}\right)$ be a bipartite minor with a maximum number of vertices of $G$ such that edges are only contracted in $G$ and multiple edges and loops are deleted in the resulting graph, if necessary. Then for any eigenfunction $f$ of a generalized Laplacian $\mathbf{M}(G), \mathfrak{W}(f) \leq\left|V^{*}\right|$.

Proof. By contraction all edges $u v$ for which $f(u), f(v) \geq 0$ and all edges $u v$ with $f(u), f(v)<0$ we get a bipartite minor of $G$. Thus every weak positive nodal domain and every strong negative nodal domain of $f$ collapses into a single vertex. This minor is bipartite and the result follows, since $G^{*}$ is bipartite minor with maximum number of vertices.

Heiko Müller [13] has remarked that finding maximal bipartite minors is also an NP-complete problem.

The upper bound based on a maximal bipartite minor does not hold for strong nodal domains. For the graph in Fig. 5.2 there exists an eigenfunction of the graph Laplacian with values $(1,-1,0,1,-1)$. Thus, it has four strong nodal domains while a maximum bipartite minor obtained by edge contractions has at most three vertices. Figure 5.3 shows an example where the maximal number of strong domains is not 


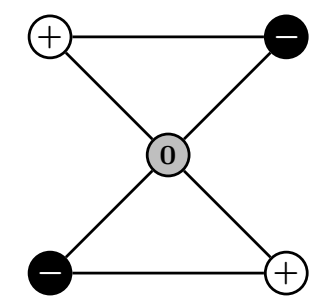

FIG. 5.2. Counterexample: the number of vertices of the maximum bipartite minor is not an upper bound for the number of strong nodal domains of an eigenfunction of a Laplacian; the eigenfunction $f$ has \& strong nodal domains but $\left|V^{*}\right|=3$.

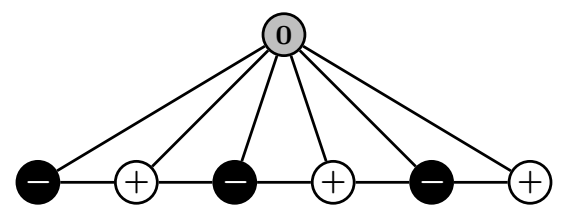

FIG. 5.3. Sign pattern of an eigenfunction $g$ with maximal number of strong nodal domains for a minor $H=G /$ uv of graph $G$ from Fig. 5.1: $\mathfrak{S}(g)=6>5=\mathfrak{S}(f)$.

even monotone for minors: There exists an eigenfunction of the Laplacian of the minor $G / u v$ with 6 strong nodal domains whereas eigenfunctions of the Laplacian of the original graph $G$ in Fig. 5.1 have at most 5 strong nodal domains.

Analogously to Thm. 5.1, one could ask whether the upper bound in Thm. 5.4 is sharp. Again the graph in Fig. 5.1 serves as a counterexample for the graph Laplacian $\mathbf{L}$, as every eigenfunction has at most 5 weak nodal domains but there exists a bipartite minor $G^{*}$ with 6 vertices. However, it is an open question whether this bound is sharp for generalized Laplacians.

Problem 5.5. Let $G^{*}$ be a maximum bipartite minor of a graph $G$ as defined in Thm. 5.4. Is there a generalized Laplacian matrix $\mathbf{M}(G)$ such that an eigenfunction of $M(G)$ has $\left|V\left(G^{*}\right)\right|$ weak nodal domains?

Acknowledgments. This work as supported in part by the German $D F G$ Bioinformatics Initiative project no. BIZ-6/1-2.

\section{REFERENCES}

[1] T. Biylkoğlu. A discrete nodal domain theorem for trees. Lin. Algebra Appl., 360:197-205, 2003.

[2] T. Bıyıkoğlu, W. Hordijk, J. Leydold, T. Pisanski, and P. F. Stadler. Graph Laplacians, nodal domains, and hyperplane arrangements. Lin. Algebra Appl., 390:155-174, 2004.

[3] T. Biylkoğlu and J. Leydold. Faber-Krahn type inequalities for trees, 2004. submitted to J. Comb. Series B.

[4] I. Chavel. Eigenvalues in Riemannian Geometry. Academic Press, Orlando Fl., 1984.

[5] F. R. K. Chung. Spectral Graph Theory, volume 92 of CBMS. American Mathematical Society, Providence RI, 1997.

[6] Y. Colin de Verdière. Multiplicités des valeurs propres Laplaciens discrets et laplaciens continus. Rendiconti di Matematica, 13:433-460, 1993. (French). 
[7] R. Courant and D. Hilbert. Methods of Mathematical Physics, Vol.1. Interscience, New York, 1953.

[8] E. B. Davies, G. M. L. Gladwell, J. Leydold, and P. F. Stadler. Discrete nodal domain theorems. Lin. Algebra Appl., 336:51-60, 2001.

[9] M. Fiedler. Eigenvectors of acyclic matrices. Czechoslovak Math. J., 25:607-618, 1975.

[10] J. Friedman. Some geometric aspects of graphs and their eigenfunctions. Duke Math. J., 69(3):487-525, March 1993.

[11] M. R. Garey and D. S. Johnson. Computers and Intractability. A Guide to the Theory of NP-Completeness. W. H. Freeman and Company, San Francisco, 1979.

[12] B. Mohar. Some applications of Laplace eigenvalues of graphs. In G. Hahn and G. Sabidussi, editors, Graph Symmetry: Algebraic Methods and Applications, volume 497 of NATO ASI Series C, pages 227-275. Kluwer, Dordrecht, 1997.

[13] H. Müller. private communication, 2001.

[14] R. Roth. On the eigenvectors belonging to the minimum eigenvalue of an essentially nonnegative symmetric matrix with bipartite graph. Lin. Algebra Appl., 118:1-10, 1989. 